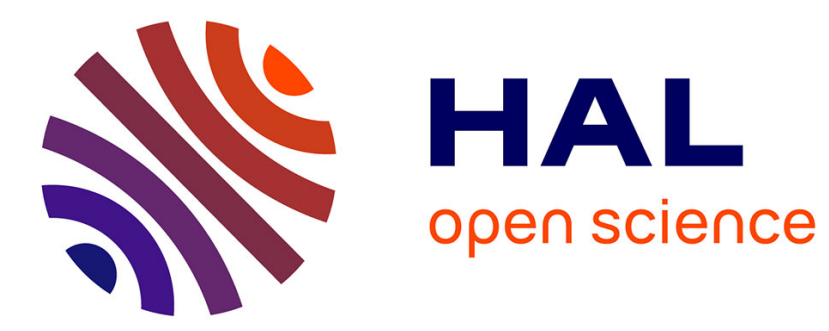

\title{
Thermodynamic Contribution of Water in Cryptophane Host-Guest Binding Reaction
}

Daryl K Eggers, Sherry Fu, Dominic V Ngo, Elizabeth H Vuong, T. Brotin

\section{To cite this version:}

Daryl K Eggers, Sherry Fu, Dominic V Ngo, Elizabeth H Vuong, T. Brotin. Thermodynamic Contribution of Water in Cryptophane Host-Guest Binding Reaction. Journal of Physical Chemistry B, 2020, 124 (30), pp.6585-6591. 10.1021/acs.jpcb.0c05354 . hal-03343470

\author{
HAL Id: hal-03343470 \\ https://hal.science/hal-03343470
}

Submitted on 14 Sep 2021

HAL is a multi-disciplinary open access archive for the deposit and dissemination of scientific research documents, whether they are published or not. The documents may come from teaching and research institutions in France or abroad, or from public or private research centers.
L'archive ouverte pluridisciplinaire HAL, est destinée au dépôt et à la diffusion de documents scientifiques de niveau recherche, publiés ou non, émanant des établissements d'enseignement et de recherche français ou étrangers, des laboratoires publics ou privés. 


\title{
Thermodynamic Contribution of Water in
}

\section{Cryptophane Host-Guest Binding Reaction}

Daryl K. Eggers, ${ }^{*, \dagger}$ Sherry Fu,,${ }^{\dagger}$ Dominic V. Ngo, Elizabeth H. Vuong, ${ }^{\dagger}$ and Thierry Brotin $*, \sharp$

† Department of Chemistry, San José State University, San José, CA 95192-0101, USA

¥Univ. Lyon, Ens de Lyon, CNRS UMR 5182, Université Claude Bernard Lyon 1, Laboratoire de Chimie, F69342, Lyon, France

\section{Corresponding Authors}

*Email: daryl.eggers@sjsu.edu, Tel: 408-924-4960

*Email: thierry.brotin@ens-lyon.fr.

\begin{abstract}
A detailed examination of binding thermodynamics is undertaken for the interaction between rubidium ion and a water-soluble cryptophane molecule using isothermal titration calorimetry. The equilibrium binding quotient for this host-guest pair decreases with increasing product formation.
\end{abstract}


When analyzed with a thermodynamic framework that considers water explicitly in the governing equation, the shift in equilibrium is interpreted as an unfavorable change in the free energy of solvation upon formation of the inclusion complex. A van't Hoff analysis of the binding data, as well as an observation of aggregation between inclusion complexes, suggests that charge-charge interactions between rubidium ion and the phenolate groups of the cryptophane host provide the driving force for association in water that overcomes a large and unfavorable change in solvent enthalpy.

KEYWORDS: Hydration, solvation, isothermal titration calorimetry. 


\section{INTRODUCTION}

Defining the forces that govern molecular interactions is fundamental to many fields of science, including molecular biology and therapeutics. Unfortunately, the role of solvent molecules in binding equilibria is not addressed clearly by the classical equations of thermodynamics.

Currently, the same equations used for gas-phase binding reactions are applied to reactions in a condensed phase. A major barrier to our full understanding of binding phenomena in solution is the inability to dissect the energetic contribution of the solvent from the overall reaction thermodynamics using experimentally-accessible approaches.

Recently, a thermodynamic framework was proposed that includes an explicit term for the change in free energy of the solvent when two molecules form a complex..$^{1}$ The governing equation predicts that the equilibrium quotient $(K)$ is not a constant with concentration when the contribution of solvent is significant; the observed binding affinity can increase or decrease with reactant concentration, depending on whether the change in solvation energy is favorable or unfavorable. This approach has been applied to the chelation of calcium ion by EDTA in water ${ }^{1}$ and to the formation of lanthanide-container complexes with various ligands in organic solvent., ${ }^{2,3}$

In the current study, the solvent-explicit framework is tested against data obtained for a hostguest reaction that employs a water-soluble cryptophane, compound 1, with rubidium ion (Figure 1). Synthetic cryptophanes are comprised of a family of spherical host molecules with a central cavity formed by two cyclotribenzylene caps and three linkers. ${ }^{4}$ The binding affinities of host $\mathbf{1}$ with $\mathrm{Cs}^{+}, \mathrm{Tl}^{+}$, and $\mathrm{Rb}^{+}$have been investigated previously at a single concentration; 5,6 the reaction selected for this study is known to be amenable to measurements by isothermal titration calorimetry (ITC) without using a competitive inhibitor. 


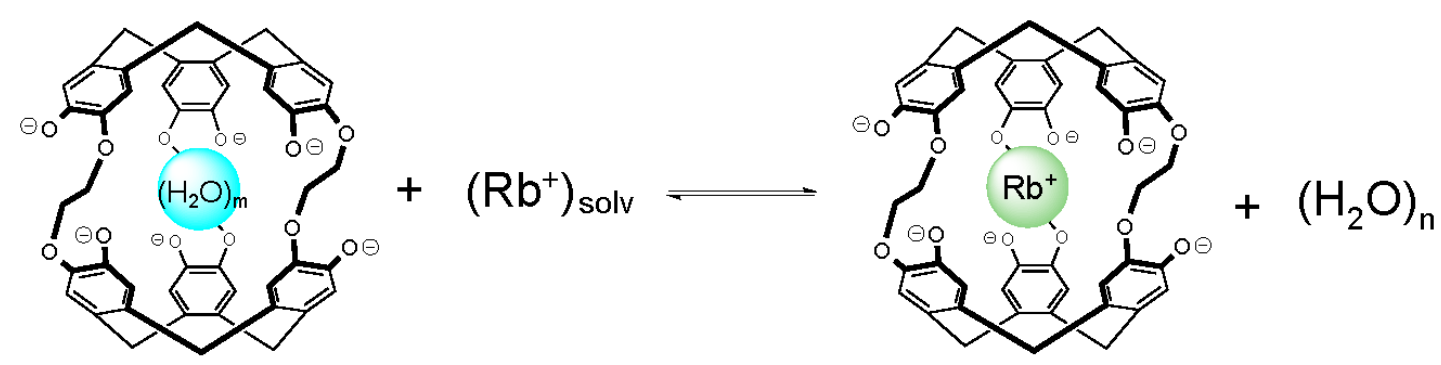

Figure 1. The binding of rubidium ion to host $\mathbf{1}$ (left) is accompanied by the displacement of water molecules from the central cavity and the hydration shell of rubidium, indicated as $\left(\mathrm{H}_{2} \mathrm{O}\right)_{n}$. Aqueous solubility of host $\mathbf{1}$ is dependent on the phenolate anions, as achieved in basic solutions.

One might expect this model binding reaction to have a significant energetic contribution from the solvent due to the presence of water molecules inside the cavity that must be displaced upon binding a guest molecule. It is known that confined water has altered physical properties relative to the unconfined state, ${ }^{7,8}$ and, consistent with this thinking, molecular simulations indicate that the binding affinity of xenon to six cryptophane variants is correlated with the average number of water molecules that must be displaced from the host cavity. ${ }^{9}$

THEORETICAL METHODS

Derivation of Governing Equation 
The generalized thermodynamic framework is given here, with minor modifications from its first introduction by Castellano and Eggers in 2013. ${ }^{1}$ The governing equation is developed from the following hypothetical binding reaction:

$$
\mathbf{P}+\mathbf{L}+n \mathbf{H}_{2} \mathbf{O}^{\text {before }} \rightleftharpoons \mathbf{Q}+n \mathbf{H}_{2} \mathbf{O}^{\text {after }}
$$

where $\mathrm{P}$ represents a protein, receptor, or host molecule, $\mathrm{L}$ denotes a specific ligand, binding partner, or guest molecule, and Q represents a 1:1 complex between the two reactants (P:L). The notations $n \mathrm{H}_{2} \mathrm{O}^{\text {before }}$ and $n \mathrm{H}_{2} \mathrm{O}^{\text {after }}$ refer to the subpopulation of " $n$ " water molecules in the reactant solvation shells that undergo a change in energy state, before and after formation of a single molecule of complex Q. This energy change includes the combined effects of (1) the rearrangement of water molecules within the solvation spheres, and (2) the release of water molecules from a solvation sphere to the bulk phase. For a specific solution of defined buffers and co-solutes (denoted by subscript $i$ ), the change in free energy of the binding reaction may be expressed as the difference in chemical potentials, $\mu_{i}$. When the solvent is included as a component of the balanced equation, we obtain the following expression for products minus reactants:

$$
\Delta G_{i}^{r x n}=\mu_{i}^{Q}+N \bar{\mu}_{i}^{\text {after }}-\left(\mu_{i}^{P}+\mu_{i}^{L}+N \bar{\mu}_{i}^{\text {before }}\right)
$$

or, rearranging,

$$
\Delta G_{i}^{r x n}=\mu_{i}^{Q}-\mu_{i}^{P}-\mu_{i}^{L}+N\left(\bar{\mu}_{i}^{\text {after }}-\bar{\mu}_{i}^{\text {before }}\right)
$$

where $N$ represents the total number of water molecules involved in the equilibrium, and where $\bar{\mu}_{i}^{a f t e r}$ and $\bar{\mu}_{i}^{\text {before }}$ refer to the average chemical potentials (per water molecule) of the solvent 
subpopulation that experiences a change in energy. The bar above each water potential is a reminder that these refer to a specific subset of water molecules and that these terms are independent of the overall concentration of water in the solution. For the reactants P, L and Q, it is customary to substitute the following expression for their chemical potentials:

$$
\mu_{i}^{x}=\mu^{\circ, x}+R T \ln a_{i}^{x}
$$

where $\mu^{o, x}$ is the standard state potential of component $x$, and where $a_{i}^{x}$ is the relative activity of $x$ in solution $i$. The activity term in eq 3 may be replaced by a concentration term using the following relationship:

$$
a_{i}^{x}=\gamma_{i}^{x}\left(\frac{[x]_{i}}{[x]^{\circ}}\right)
$$

where $\gamma_{i}^{x}$ is the activity coefficient, $[x]_{i}$ denotes the concentration of species $x$, and $[x]^{\circ}$ denotes the reference concentration of species $x$. In the framework presented here, the activity coefficients are viewed as unnecessary $\left(\gamma_{i}^{x}=1\right)$ because, under most conditions, the explicit treatment of water in eq 2 will account for "nonideal" behavior. Experimental observations that deviate from the classical equation are viewed as arising from solute-solvent interactions, as opposed to solutesolute interactions, and the contributions of solute-solvent interactions are conveyed by the solvation terms, $\bar{\mu}_{i}^{\text {after }}$ and $\bar{\mu}_{i}^{\text {before }}$. Substituting eq 4 into eq 3 and omitting the activity coefficients, we obtain the following: 


$$
\mu_{i}^{x}=\mu^{\circ, x}+R T \ln [x]_{i}-R T \ln [x]^{\circ}
$$

Further substitution of eq 5 into eq 2 for components $\mathrm{P}, \mathrm{L}$ and $\mathrm{Q}$, leads to the following expression:

$$
\Delta G_{i}^{r x n}=\left(\mu_{i}^{\circ, Q}-\mu_{i}^{o, P}-\mu_{i}^{\circ, L}\right)+R T \ln \frac{[Q]_{i}}{[P]_{i}[L]_{i}}-R T \ln \frac{[Q]^{\circ}}{[P]^{\circ}[L]^{\circ}}+N\left(\bar{\mu}_{i}^{\text {after }}-\bar{\mu}_{i}^{\text {before }}\right)
$$

Next, the three standard state potentials and the reference concentration term may be combined into one constant, a (modified) standard state free energy:

$$
\left(\mu_{i}^{\circ, Q}-\mu_{i}^{\circ, P}-\mu_{i}^{\circ, L}\right)-R T \ln \frac{[Q]^{\circ}}{[P]^{\circ}[L]^{\circ}}=\Delta G^{\circ}
$$

Substituting eq 7 into eq 6 yields:

$$
\Delta G_{i}^{r x n}=R T \ln \frac{[Q]_{i}}{[P]_{i}[L]_{i}}+\Delta G^{\circ}+N\left(\bar{\mu}_{i}^{\text {after }}-\bar{\mu}_{i}^{\text {before }}\right)
$$

The solvent potentials that appear in eq 8 require special consideration. Because the chemical potential of water is a complicated function of hydrogen-bond strength and molecular orientation and not a strong function of the water concentration or number density, eq 3 is not an appropriate substitution for the solvent potentials. As an alternative, the chemical potentials of water are expressed as free energy values using the following relationship:

$$
\mu_{i}^{H 2 O}=\bar{g}^{\circ}+\bar{g}_{i}^{H 2 O}
$$


where $\bar{g}^{\circ}$ is the standard state free energy of water per mole of water, and where $\bar{g}_{i}^{H 2 O}$ denotes the free energy per mole of a specific subset of water molecules (before or after complex formation in solution $i$ ). Note that the standard state free energy of water will cancel out when eq 9 is substituted into eq 8 for the two solvation terms because water is treated as both a reactant and product of the balanced reaction. The total number of participating water molecules per reaction volume, $N$, is related by stoichiometry to the product concentration as follows:

$$
N=n[Q]_{i}
$$

where, once again, $n$ is the number of water molecules in the reactant solvation shells that change energy state on formation of a single molecule of complex Q. Substituting eq 9 and eq 10 into eq 8 leads to the following expression:

$$
\Delta G_{i}^{r x n}=R T \ln \frac{[Q]_{i}}{[P]_{i}[L]_{i}}+\Delta G^{\circ}+n[Q]_{i}\left(\bar{g}_{i}^{\text {after }}-\bar{g}_{i}^{\text {before }}\right)
$$

Equation 11 may be simplified further by defining the change in solvation energy, $\Delta G_{i}^{H 2 O}$ :

$$
\Delta G_{i}^{H 2 O}=n\left(\bar{g}_{i}^{\text {after }}-\bar{g}_{i}^{\text {before }}\right)
$$

leading to a general expression for binding reactions in aqueous solution: 


$$
\Delta G_{i}^{r x n}=R T \ln \frac{[Q]_{i}}{[P]_{i}[L]_{i}}+[Q]_{i} \Delta G_{i}^{H 2 O}+\Delta G^{\circ}
$$

At equilibrium, $\Delta G_{i}^{r x n}=0$, and substitution into eq 13 leads to the following:

$$
\Delta G^{\circ}=-R T \ln \frac{[Q]_{i}^{e q}}{[P]_{i}^{e q}[L]_{i}^{e q}}-[Q]_{i}^{e q} \Delta G_{i}^{H 2 O}
$$

where $[x]_{i}^{e q}$ denotes the observed concentration of components $\mathrm{P}, \mathrm{L}$ and $\mathrm{Q}$ at equilibrium in solution $i$. Following tradition, the equilibrium concentrations within the natural logarithm term may be replaced by the equilibrium quotient, $K_{i}$, yielding the final governing equation for binding reactions at equilibrium:

$$
\Delta G^{\circ}=-R T \ln K_{i}-[Q]_{i}^{e q} \Delta G_{i}^{H 2 O}
$$

Notes on Thermodynamic Framework

(a) In practical application of eqs 4-15, all concentrations are treated as dimensionless quantities but must be expressed in the same units, typically molarity in the field of biochemistry. Thus, the magnitudes of the free energy values are dependent on the definition of the standard state which reflects the choice of concentration units.

(b) As pointed out previously by Castellano and Eggers, ${ }^{1}$ when all concentrations in eq 13 are unity, $\Delta G_{i}^{r \times n}=\Delta G_{i}^{H 2 O}+\Delta G^{\circ}$. It follows that the relationship, $\Delta G_{i}^{r x n}=\Delta G^{\circ}$, is satisfied only 
when the reaction quotient within the natural logarithm term in eq 13 is unity and when the product concentration $[\mathrm{Q}]$ approaches zero. Thus, it no longer seems necessary to maintain the confounding definition of the reference concentration as a 1.0 molal or 1.0 molar solution that behaves "as if infinitely dilute." Instead, an infinitely dilute solution should be adopted as the reference state if one wants the reference state to reflect the condition where $\Delta G_{i}^{r x n}$ and $\Delta G^{\circ}$ are equivalent.

(c) It is preferable to refer to $K_{i}$ as the equilibrium quotient - and not as the equilibrium constant because, as demonstrated in this work, $K_{i}$ is expected to vary with product concentration.

(d) The subscript " $i$ " in the notation for $K_{i}$ and $\Delta G_{i}^{H 2 O}$ is retained in the general equations to denote that the value of each parameter is dependent on the specific solution conditions in which the measurements were performed; the average free energy of bulk water contributes to the value of $\bar{g}_{i}^{a f t e r}$ in eq 12 if any water molecules are released from the solvation spheres to the bulk phase on complex formation. The average free energy of bulk water depends on all species in the solution, including buffers and secondary solutes that do not participate directly in the reaction, because these solutes also have a solvation shell that varies in energy from water distant from their surface. (e) Related to item d above, the governing equation at equilibrium (eq 15) assumes that secondary solutes do not bind with any significant affinity to the reactants $(P, L, Q)$, such that the number of water molecules in contact with the binding reactants far outnumber the cosolutes in contact with the reactants. This assumption is contrary to the theory of preferential interactions that views all changes in binding equilibria as a result of differential contacts between the secondary solutes and the reactants, while ignoring the contribution of water. The theory of preferential interactions does not address observations like those in the current report for which the binding equilibrium shifts in response to changes in reactant concentration as the concentration of secondary solutes is held constant. 
(f) Though eq 15 was derived for aqueous reactions, this equation may be applicable to binding reactions in any solvent, as demonstrated by Piguet and coworkers. ${ }^{2}$ The notation for $\Delta G_{i}^{H 2 O}$ may be replaced with the generic term $\Delta G_{i}^{\text {solv }}$ in the governing equations when water is not the reaction solvent.

\section{EXPERIMENTAL METHODS}

\section{Reagents}

Compound 1 was prepared according to a published procedure with careful attention to the purification steps..$^{10}$ The proton NMR of the purified host is given in the Supporting Information, Fig. S1. Rubidium chloride $(99+\%)$ and sodium hydroxide were obtained from Fisher Scientific. Stock solutions were made at room temperature using ultrapure water (Millipore, Milli-Q system). All solutions contained 0.20 M sodium hydroxide $(\mathrm{pH}>13)$ to ensure that the phenol groups of cryptophane remain in the deprotonated state. Cryptophane solutions were made fresh and used on the same day as each binding experiment.

\section{ITC Experiments}

Isothermal titration calorimetry was performed with a Microcal instrument, model VP-ITC, using the analysis programs provided by the manufacturer (Origin software). All solutions were degassed under vacuum (ThermoVac). Prior to each calorimetry run, the sample cell (1449 $\mu \mathrm{L}$ volume) was cleaned and rinsed with one volume of $0.20 \mathrm{M} \mathrm{NaOH}$ prior to loading a cryptophane solution of desired concentration. The injection syringe $(\sim 300 \mu \mathrm{L})$ was filled with $\mathrm{RbCl}$ at a concentration 10-fold higher than the host concentration in the sample cell. The analog 
input range and reference power settings were adjusted in accord with the sample concentrations and expected peak output for a given run, as given in Table S1. In all trials, the first injection was set at $2 \mu \mathrm{L}$ and the first titration peak was discarded from the analysis due to the unavoidable error in the actual injection volume after filling and transfer of the syringe. For each $\mathrm{RbCl}$ concentration utilized, reference data were obtained by injecting $\mathrm{RbCl}$ into $0.20 \mathrm{M} \mathrm{NaOH}$, and the recorded enthalpies were subtracted from the corresponding binding experiments prior to regression analysis.

\section{RESULTS}

The binding experiments for this study were designed for analysis against eq 15, as motivated by the relationship first proposed by Castellano and Eggers. ${ }^{1}$ Referring to eq $15, K_{\mathrm{a}}$ is the observed equilibrium quotient in the direction of association, $[Q]_{i}$ is the equilibrium concentration of the complex, and $\Delta G_{i}^{H 2 O}$ is the corresponding change in solvation free energy in a defined solution "i." Assuming $\Delta G^{\circ}$ and $\Delta G^{\mathrm{H} 2 \mathrm{O}}$ are constants for a given reaction pair in the concentration range examined, an increase in complex formation will increase the contribution of water as a direct multiplier of the solvation energy in eq 15 and, consequently, will lead to a corresponding change in the value of $K_{\mathrm{a}}$, the one remaining variable. At infinite dilution, the last term in eq 15 becomes negligible, and the governing relationship is reduced to the classical equation found in all textbooks. In the millimolar range of product formation, however, the solvent contribution will become apparent if the absolute value of $\Delta G^{\mathrm{H} 2 \mathrm{O}}$ is far from zero.

It should be noted that $\Delta G^{\mathrm{H} 2 \mathrm{O}}$ represents the sum of at least two energetic terms, one due to displacement of water from the host cavity and the guest ion shell (desolvation) and another term 
due to rearrangement of water on the outer surface of the host following complex formation. For this reason, we find it preferable to refer to $\Delta G^{\mathrm{H} 2 \mathrm{O}}$ as the change in solvation energy (or change in hydration energy), as opposed to the more-exclusive "desolvation energy."

The binding behavior of $\mathrm{Rb}^{+}$with compound $\mathbf{1}$ was examined as a function of concentration while keeping the molar ratio of guest to host in a constant range. All solutions contained $0.2 \mathrm{M}$ $\mathrm{NaOH}$ to maximize host solubility and to maintain a constant ionic strength. Isothermal titration calorimetry data were obtained at four concentrations up to $3 \mathrm{mM}$ and at four temperatures up to $323 \mathrm{~K}$. For all ITC runs, the calorimeter cell was loaded with the desired concentration of the cryptophane host, and the syringe was loaded with $\mathrm{RbCl}$ at a 10-fold higher concentration such that the molar ratio of $\mathrm{Rb}^{+}$to host approached $2: 1$ near the end of the titration.

A small but significant change in the equilibrium quotient was observed as a function of concentration for the lower three temperatures of 283, 298, and $310 \mathrm{~K}$ (Table 1). Attempts to expand the data to include higher concentrations were unsuccessful due to an aggregation phenomenon between inclusion complexes. The unbound host was soluble to $10 \mathrm{mM}$ concentration in basic solutions, but the complex with rubidium was observed to aggregate at concentrations above $2 \mathrm{mM}$ at $283 \mathrm{~K}$ and above $3 \mathrm{mM}$ at $298 \mathrm{~K}$. Aggregation was apparent to the eye as a cloudy solution when removed from the calorimeter cell after a completed trial. It is important to note that the aggregation phenomenon yields abnormal titration profiles and results in misleadingly high $K_{\mathrm{a}}$ values if ignored (see Figure S2 for an example).

Table 1. Equilibrium Quotients as a Function of Concentration and Temperature ${ }^{\mathrm{a}, \mathrm{b}}$ 


\begin{tabular}{ccccc}
$\begin{array}{c}\text { Initial } \\
\text { [host 1] in } \\
\text { cell }(\mathrm{mM})\end{array}$ & $\begin{array}{c}{[\mathrm{RbCl}]} \\
\text { at } 1: 1 \text { ratio } \\
(\mathrm{mM})\end{array}$ & \multicolumn{3}{c}{$\begin{array}{c}\text { Equilibrium Quotient, } K_{a} \\
\left(10^{5} \pm \text { error }\right)\end{array}$} \\
0.10 & 0.087 & $11.36 \pm 0.29$ & $\mathrm{~T}=298 \mathrm{~K}$ & $\mathrm{~T}=310 \mathrm{~K}$ \\
1.00 & 0.870 & $8.12 \pm 0.70$ & $6.02 \pm 0.08$ & $4.29 \pm 0.36$ \\
2.00 & 1.80 & $7.72 \pm 0.78$ & - & - \\
3.00 & 2.70 & - & $4.55 \pm 0.14$ & $3.46 \pm 0.13$
\end{tabular}

${ }^{\text {a }}$ All solutions contain $0.2 \mathrm{M} \mathrm{NaOH}$. ${ }^{\text {b }}$ The uncertainty in each $K$ value is based on the error of precision from four or more trials.

The measured equilibrium quotients were analyzed against eq 15 by plotting $-R T \ln K_{\mathrm{a}}$ versus the complex concentration (Figure 2). The concentration of $\mathrm{RbCl}$ in the cell, just prior to the injection that surpasses a 1:1 molar ratio of guest to host, was used as the effective concentration of complex for each titration. ${ }^{1}$ Although the equilibrium is expected to shift slightly with each injection, the value of $K_{\mathrm{a}}$ obtained from curve fitting is highly dependent on the titration points that just precede attainment of a 1:1 ratio (corresponding to the steepest rise in the titration curve), and, for equilibria with association binding quotients above $10^{5}$, the concentration of complex is nearly equal to the total amount of $\mathrm{Rb}^{+}$in the cell at this point in the titration. The linear correlation observed in Figure 2 at each temperature, as suggested by eq 15, is weakest at $283 \mathrm{~K}$, the data set with the most restricted concentration range due to aggregation of the inclusion complex. At the highest temperature of $323 \mathrm{~K}$, the data were found to be independent of concentration up to $5 \mathrm{mM}$; all $K_{\mathrm{a}}$ values were within the error of precision at this temperature, represented by a horizontal line in Figure 2. The resulting free energy values from this analysis are summarized in Table 2. 


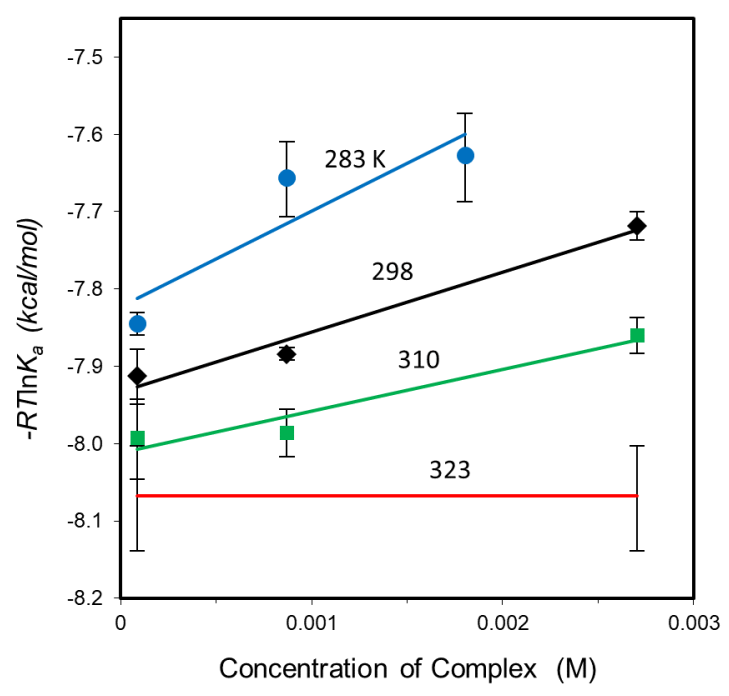

Figure 2. Linear binding relationship in accord with eq 15. Slope and $y$-intercept represent $\Delta G^{\circ}$ and $\Delta G^{\mathrm{H} 2 \mathrm{O}}$, as reported in Table 2. The lower red line was calculated from the average value of $K_{\mathrm{a}}$ obtained for all concentrations and trials at $\mathrm{T}=323 \mathrm{~K}\left(K_{\mathrm{a}}=2.86 \pm 0.30\right)$. Error bars reflect the uncertainty in $K_{\mathrm{a}}$.

Table 2. Free Energies Derived from Application of Equation 15

$\begin{array}{ccc}\mathrm{T} & \Delta G^{\circ} & \Delta G^{\mathrm{H} 2 \mathrm{O}} \\ (\mathrm{K}) & (\mathrm{kcal} / \mathrm{mol}) & (\mathrm{kcal} / \mathrm{mol})^{\mathrm{a}} \\ 283 & -7.8 & 120 \\ 298 & -7.9 & 77 \\ 310 & -8.0 & 54 \\ 323 & -8.1 & \sim 0\end{array}$

${ }^{a}$ Hydration free energy units are per mole of complex formed.

Further information may be gleaned from a van’t Hoff analysis of the free energies at all temperatures (Figure 3). The standard state free energies yield enthalpy and entropy values of $\Delta H^{\circ}=-6.1 \mathrm{kcal} / \mathrm{mol}$ and $\Delta S^{\circ}=+0.0061 \mathrm{kcal} / \mathrm{mol} \cdot \mathrm{K}$, and the change in solvation free energies 
yield $\Delta H^{\mathrm{H} 2 \mathrm{O}}=+970 \mathrm{kcal} / \mathrm{mol}$ and $\Delta S^{\mathrm{H} 2 \mathrm{O}}=+3.0 \mathrm{kcal} / \mathrm{mol} \cdot \mathrm{K}$. The enthalpy of binding as measured directly by the ITC is about $-5.5 \mathrm{kcal} / \mathrm{mol}$ at $298 \mathrm{~K}$ (Table S2).

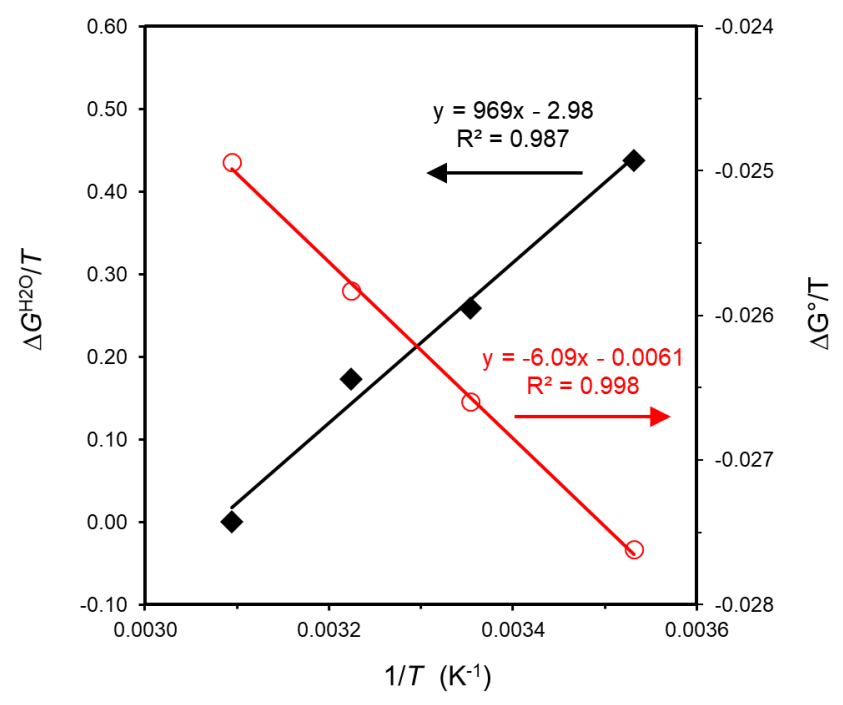

Figure 3. A van't Hoff plot of the free energies derived from eq 15 at each temperature. The enthalpy and entropy values correspond to the slope and $y$-intercept of each line, respectively. Arrows point toward the corresponding axis.

\section{DISCUSSION}

Classical thermodynamic equations view the equilibrium quotient as a constant, but such treatment may not be adequate for reactions in a condensed phase where the solvent plays an active role, as demonstrated here for a model host-guest binding reaction in water. In essence, the new thermodynamic framework employed here attributes deviations from the classical equation (nonideal behavior) to the contribution of solvent-reactant and solvent-solvent 
interactions. The $\Delta G^{\mathrm{H} 2 \mathrm{O}}$ term replaces the need for an activity coefficient, an ill-defined parameter from classical thermodynamics that provides no insight on solvent contributions.

The unfavorable change in solvation free energy for binding of $\mathrm{Rb}^{+}$to host $\mathbf{1}$ was not anticipated at the beginning of the study, but this finding is consistent with other results. Namely, the observation that the inclusion complex is more susceptible to aggregation than the unbound host suggests that the phenolate anions are in contact with $\mathrm{Rb}^{+}$in the inclusion complex, thereby decreasing the net charge of the complex, reducing the strength of water interactions, and lowering solubility. The loss of rubidium-water and phenolate-water interactions to accommodate rubidium-phenolate interactions within the host cavity should contribute to an unfavorable change in the free energy of hydration, as observed here from analysis of experimental data (Table 2). A computational study further elucidates the importance of electrostatic forces in cryptophane-guest interactions. ${ }^{11}$

The magnitudes of the $\Delta G^{\mathrm{H} 2 \mathrm{O}}$ values are large relative to the standard state free energies (Table 2), but these values are reasonable when compared to the thermodynamic data tabulated by Marcus for the transfer of ions from the ideal gas phase to an infinitely dilute aqueous solution. ${ }^{12}$ For example, the free energy of hydration for the rubidium ion is reported to be -67 $\mathrm{kcal} / \mathrm{mol}$. The phenolate anion is not included in the Marcus tables, but another aromatic oxyanion, picrate, is listed with a free energy of hydration of $-47 \mathrm{kcal} / \mathrm{mol}$. Thus, assuming the rubidium ion is fully desolvated in the inclusion complex and one or more phenolate groups are partially desolvated in the complex, the sign and magnitude of the $\Delta G^{\mathrm{H} 2 \mathrm{O}}$ values in Table 2 are justified. It should be noted that all hydration energies reported here are given in units per mole of complex formed, as opposed to units per mole of water. 
The values of $\Delta H^{\mathrm{H} 2 \mathrm{O}}$ and $\Delta S^{\mathrm{H} 2 \mathrm{O}}$ obtained from the van't Hoff analysis are more difficult to comprehend. In the case of $\Delta H^{\mathrm{H} 2 \mathrm{O}}$, the large enthalpy change ( $\left.+970 \mathrm{kcal} / \mathrm{mol}\right)$ may be viewed as further indication that strong water-phenolate interactions are lost or weakened, concurrent with a loss of water- $\mathrm{Rb}^{+}$interactions, upon formation of the inclusion complex. This conclusion is supported by a computational study with another host-guest system that employed molecular dynamics simulations on a microsecond time scale..$^{13}$ Through precise calculations of binding enthalpy for cucurbit[7]uril with eight guest molecules, Gilson and coworkers found the change in hydration enthalpy was large and unfavorable due to the loss of solute-water interactions upon binding. The magnitude of the hydration enthalpy was found to increase with the electrostatic character of the solutes, and the values ranged from +163 to $+347 \mathrm{kcal} / \mathrm{mol}$ for binding of the host with the charged guest molecules..$^{13}$ The unfavorable loss of water-solute interactions, combined into one term for guest and host, was partially compensated by the gain in water-water interactions. Because binding of rubidium ion to host $\mathbf{1}$ may involve the participation of six negative charges in addition to possible cation-pi interactions, it is reasonable to expect $\Delta H^{\mathrm{H} 2 O}$ for this system to be larger in magnitude than the enthalpies reported for the cucurbituril system. As articulated by Gilson and coworkers, "binding enthalpies are delicate balances of much larger energetic shifts in solute-solute, solute-solvent, and solvent-solvent interactions.”13

In the case of $\Delta S^{\mathrm{H} 2 \mathrm{O}}$, a positive entropy change is expected due to the release of water molecules to the bulk phase upon formation of the inclusion complex, but an entropy change of $+3 \mathrm{kcal} / \mathrm{mol} \cdot \mathrm{K}$ seems to be high by an order of magnitude. Scanning the extensive list of entropies for hydrated ions, as tabulated by Marcus, most values are in the range of $0.02-0.10$ $\mathrm{kcal} / \mathrm{mol} \cdot \mathrm{K} \cdot{ }^{12} \mathrm{~A}$ similar range in hydration entropy has been estimated for the binding of drug molecules to cyclodextrin hosts. ${ }^{14}$ One possibility for the large hydration entropy reported here is 
that the subset of water molecules within the cavity are characterized by an intrinsically-low entropy for which there is no model system to compare; 2-3 water molecules may be trapped within the cage on a long timescale prior to binding of the guest. Another possibility is that formation of the inclusion complex is accompanied by a change in the protonation state of one or more of the six phenolate groups that line the perimeter of the host and confer solubility. All experiments were performed at $\mathrm{pH}>13$ which is well above the acid dissociation constant of $\mathrm{p} K \sim 10$ for phenol, but charge repulsion due to the proximity of the six phenolate anions to each other may lead to altered acid-base behavior. If true, binding of the $\mathrm{Rb}^{+}$guest would relieve the charge repulsion and allow a proton to be released from one or more phenol groups. The loss of a proton upon guest binding would not affect the measured binding quotients in Table 1, but a linked ionization and neutralization reaction would complicate interpretation of the (large) hydration enthalpy and entropy values reported here. The fact that the standard state binding enthalpy obtained from Figure 3 is more negative than the ITC-measured binding enthalpy by $0.6 \mathrm{kcal} / \mathrm{mol}$ supports the hypothesis that other, linked equilibria contribute to the observed thermodynamics.

Yet another factor of unknown magnitude in this analysis is the subset of sodium ions that serve as counter ions for the phenolate groups prior to guest binding; the calculated changes in hydration energy must also include any contributions due to the transfer of $\mathrm{Na}^{+}$from the surface of the host to the bulk phase when rubidium binds. The distributions of sodium ions have been investigated by simulation for cryptophane derivatives containing carboxylate capping groups in place of the phenolate anions. ${ }^{9}$ Also, the binding affinity of host $\mathbf{1}$ is known to be altered by the concentration and identity of the counter ion supplied with the strong base $\left(\mathrm{Li}^{+}, \mathrm{Na}^{+}\right.$, or $\left.\mathrm{K}^{+}\right){ }^{6}$ 
These counter ions could influence guest binding by two mechanisms, competition with $\mathrm{Rb}^{+}$and ion-dependent changes in hydration thermodynamics.

The estimated values of $\Delta H^{\mathrm{H} 2 \mathrm{O}}$ and $\Delta S^{\mathrm{H} 2 \mathrm{O}}$ for binding of rubidium ion to host $\mathbf{1}$ are about 3-fold higher than the corresponding values reported previously for binding of EDTA to calcium, another reaction where the association of oppositely-charged groups dominates over an unfavorable change in solvation free energy. ${ }^{1}$ Another example of a reaction that follows eq 15 is revealed in Figure S3 for binding of a sulfonated, glycoluril-based molecular clip with pxylenediammonium $\left(\Delta G^{\mathrm{H} 2 \mathrm{O}}=+44 \mathrm{kcal} / \mathrm{mol}\right.$ at $\left.298 \mathrm{~K}\right)$. The molecular clip pairing was included in the SAMPL5 binding prediction challenge. ${ }^{15,16}$ Thus, three model binding reactions, all involving charge-charge interactions of opposite sign, have been characterized by unfavorable changes in solvation free energy when the experimental data are analyzed with the solventexplicit framework, as implemented with eq 15.

\section{CONCLUSIONS}

Understanding the role of water in binding equilibria may be critical for developing drugs with high specificity for their intended targets, in addition to characterizing the molecular interactions that define a living cell. This work demonstrates one of few options for obtaining information on the thermodynamics of solvation from experimental binding data. The fact that $\Delta G^{\mathrm{H} 2 \mathrm{O}}$ is an order of magnitude larger than $\Delta G^{\circ}$ for this model host-guest system strongly supports the idea that binding equilibria in the condensed phase are governed by relatively small differences in large opposing forces. Future experiments with other guest-host reactions, 
including cryptophane derivatives, may elucidate further the role of water in binding equilibria as a function of surface chemistry.

\section{ASSOCIATED CONTENT}

\section{Supporting Information.}

The Supporting Information is available free of charge.

Calorimeter settings, enthalpies of binding, NMR spectrum of host 1, sample ITC runs, and molecular clip data.

\section{AUTHOR INFORMATION}

\section{Corresponding Authors}

*Email: daryl.eggers@sjsu.edu.

*Email: thierry.brotin@ens-lyon.fr.

\section{Notes}

The authors declare no competing financial interests.

\section{ACKNOWLEDGMENT}


This research was funded by a grant to D.K.E. from the National Institutes of Health (R15GM089591). T.B. gratefully acknowledges support from CNRS. The molecular clip molecule was a kind gift from L. Isaacs (U. Maryland, College Park).

\section{REFERENCES}

(1) Castellano, B. M.; Eggers, D. K. Experimental Support for a Desolvation Energy Term in Governing Equations for Binding Equilibria. J. Phys. Chem. B 2013, 117, 8180-8188.

(2) Baudet, K.; Guerra, S.; Piguet, C. Chemical Potential of the Solvent: A Crucial Player for Rationalizing Host-Guest Affinities. Chem. Eur. J. 2017, 23, 16787-16798.

(3) Baudet, K.; Kale, V.; Mirzakhani, M.; Babel, L.; Naseri, S.; Besnard, C.; Nozary, H.; Piguet, C. Neutral Heteroleptic Lanthanide Complexes for Unravelling Host-Guest Assemblies in Organic Solvents: The Law of Mass Action Revisited. Inorg. Chem. 2020, $59,62-75$.

(4) Brotin, T.; Dutasta, J.-P. Cryptophanes and Their Complexes-Present and Future. Chem. Rev. 2009, 109, 88-130.

(5) Brotin, T.; Montserret, R.; Bouchet, A.; Cavagnat, D.; Linares, M.; Buffeteau, T. High Affinity of Water-Soluble Cryptophanes for Cesium Cations. J. Org. Chem. 2012, 77, 1198-1201.

(6) Brotin, T.; Goncalves, S.; Berthault, P.; Cavagnat, D.; Buffeteau, T. Influence of the Cavity Size of Water-Soluble Cryptophanes on Their Binding Properties for Cesium and Thallium Cations. J. Phys. Chem. B 2013, 117, 12593-12601.

(7) Giovambattista, N.; Rossky, P. J.; Debenedetti, P. G. Computational Studies of Pressure, Temperature, and Surface Effects on the Structure and Thermodynamics of Confined Water. Annu. Rev. Phys. Chem. 2012, 63, 179-200. 
(8) Biedermann, F.; Nau, W. M.; Schneider, H.-J. The Hydrophobic Effect RevisitedStudies with Supramolecular Complexes Imply High-Energy Water as a Noncovalent Driving Force. Angew. Chem. Int. Ed. 2014, 53, 11158-11171.

(9) Gao, L.; Liu, W.; Lee, O.-S.; Dmochowski, I. J.; Saven, J. G. Xe Affinities of WaterSoluble Cryptophanes and the Role of Confined Water. Chem. Sci. 2015, 6, 7238-7248.

(10) Canceill, J.; Lacombe, L.; Collet; A. Water-Soluble Cryptophane Binding Lipophilic Guests in Aqueous Solution. J. Chem. Soc. Chem. Comm., 1987, 219-221.

(11) Chapellet, L.-L.; Dognon, J.-P.; Jean, M.; Vanthuyne, N.; Berthault, P.; Buffeteau, T.; Brotin, T. Experimental and Theoretical Study of the Complexation of Cesium and Thallium Cations by a Water-Soluble Cryptophane. ChemistrySelect 2017, 2, 5292-5300.

(12) Marcus, Y. Ion Properties, Marcel Dekker, New York, 1997, Chp. 8, 117-149.

(13) Fenley, A. T.; Henriksen, N. M.; Muddana, H. S.; Gilson, M. K. Bridging Calorimetry and Simulation through Precise Calculations of Cucurbituril-Guest Binding Enthalpies. J. Chem. Theory Comput. 2014, 10, 4069-4078.

(14) Chen, W.; Chang, C.-E.; Gilson, M. K. Calculation of Cyclodextrin Binding Affinities: Energy, Entropy, and Implications for Drug Design. Biophys. J. 2004, 87, 3035-3049.

(15) Yin, J.; Henriksen, N. M.; Slochower, D. R.; Shirts, M. R.; Chiu, M. W.; Mobley, D. L.; Gilson, M. K. Overview of the SAMPL5 Host-Guest Challenge: Are We Doing Better? J. Comput. Aided Mol. Des. 2017, 31, 1-19.

(16) She, N.; Moncelet, D.; Gilberg, L.; Lu, X.; Sindelar, V.; Briken, V.; Isaacs, L. GlycolurilDerived Molecular Clips are Potent and Selective Receptors for Cationic Dyes in Water. Chem. Eur. J. 2016, 22, 15270-15279. 